BMC

Plant Biology

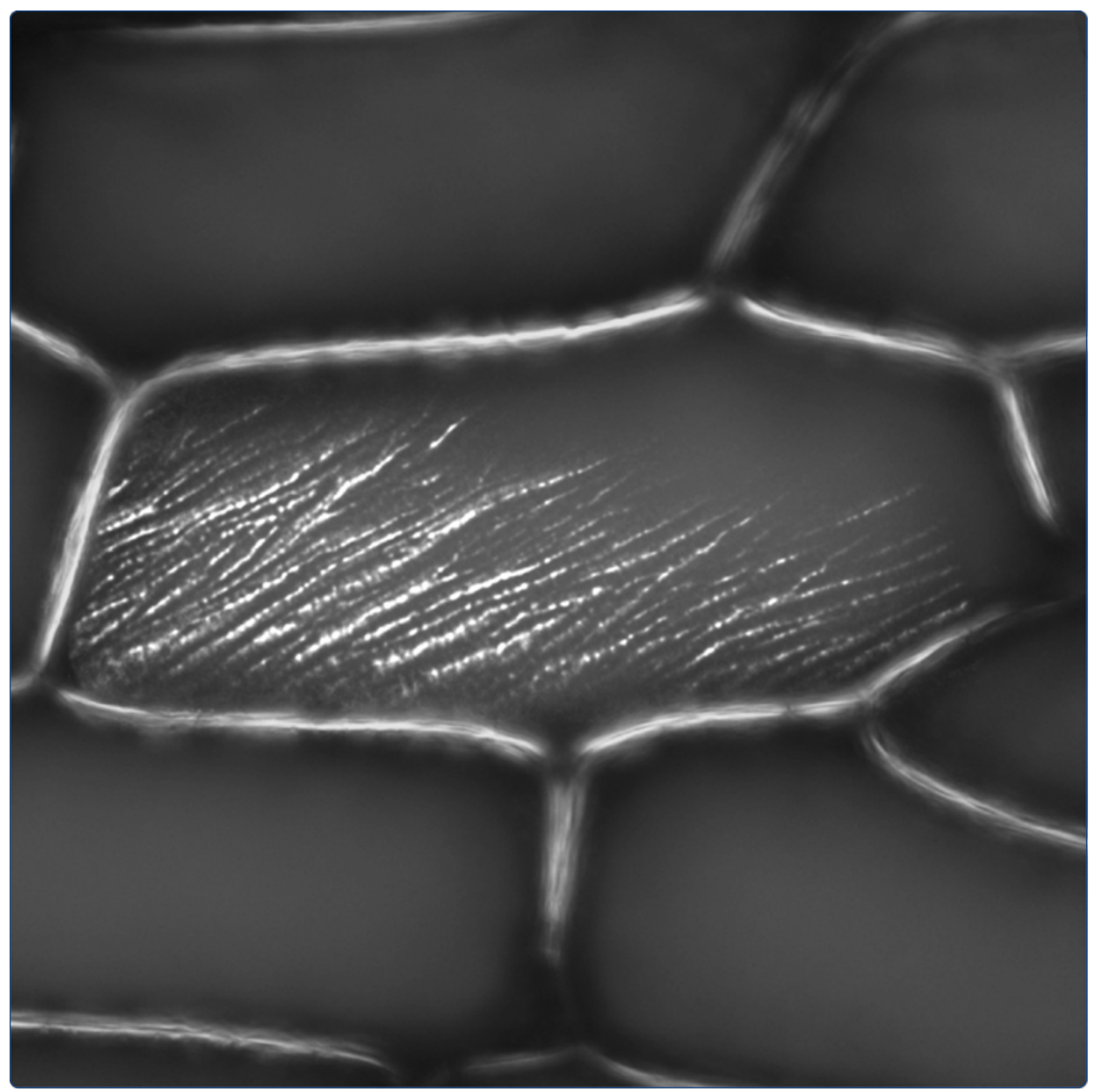

Super-resolution imaging with Pontamine Fast Scarlet 4BS enables direct visualization of cellulose orientation and cell connection architecture in onion epidermis cells

Liesche et al. 


\title{
Super-resolution imaging with Pontamine Fast Scarlet 4BS enables direct visualization of cellulose orientation and cell connection architecture in onion epidermis cells
}

\author{
Johannes Liesche*, Iwona Ziomkiewicz and Alexander Schulz
}

\begin{abstract}
Background: In plants, a complex cell wall protects cells and defines their shape. Cellulose fibrils form a multilayered network inside the cell-wall matrix that plays a direct role in controlling cell expansion. Resolving the structure of this network will allow us to comprehend the relationship of cellulose fibril orientation and growth.

The fluorescent dye Pontamine Fast Scarlet 4BS (PFS) was shown to stain cellulose with high specificity and could be used to visualize cellulose bundles in cell walls of Arabidopsis root epidermal cells with confocal microscopy. The resolution limit of confocal microscopy of some $200 \mathrm{~nm}$ in $x y$ and $550 \mathrm{~nm}$ in z for green light, restricts the direct visualization of cellulose to relatively large bundles, whereas the structure of cellulose microfibrils with their diameter below $10 \mathrm{~nm}$ remains unresolved. Over the last decade, several so-called super-resolution microscopy approaches have been developed; in this paper we explore the potential of such approaches for the direct visualization of cellulose.
\end{abstract}

Results: To ensure optimal imaging we determined the spectral properties of PFS-stained tissue. PFS was found not to affect cell viability in the onion bulb scale epidermis. We present the first super-resolution images of cellulose bundles in the plant cell wall produced by direct stochastic optical reconstruction microscopy (dSTORM) in combination with total internal reflection fluorescence (TIRF) microscopy. Since TIRF limits observation to the cell surface, we tested as alternatives 3D-structured illumination microscopy (3D-SIM) and confocal microscopy, combined with image deconvolution. Both methods offer lower resolution than STORM, but enable 3D imaging. While 3D-SIM produced strong artifacts, deconvolution gave good results. The resolution was improved over conventional confocal microscopy and the approach could be used to demonstrate differences in fibril orientation in different layers of the cell wall as well as particular cellulose fortifications around plasmodesmata.

Conclusions: Super-resolution light microscopy of PFS-stained cellulose fibrils is possible and the increased resolution over conventional approaches makes it a valuable tool for the investigation of the cell-wall structure. This is one step in method developments that will close the gap to more invasive techniques, such as atomic force and electron microscopy.

Keywords: Fluorescent dye, Cell wall, Cellulose, STORM, Structured illumination, Super-resolution microscopy, TIRF, Deconvolution

\footnotetext{
* Correspondence: joli@life.ku.dk

Department of Plant and Environmental Sciences, University of Copenhagen,

Thorvaldsensvej 40, Frederiksberg 1871, Denmark
} 


\section{Background}

Cellulose microfibrils form the backbone of the complex cell wall of plant cells. The exact structure of the cellulose fibril network has important implications for our understanding of plant growth on a cellular level, as it has a direct role in controlling cell elongation [1-3]. In addition, precise knowledge of cell wall architecture facilitates the development of methods for efficient breakdown of plant cell walls in the production of biofuels $[4,5]$.

Pontamine Fast Scarlet 4BS (PFS), a fluorescent dye that binds cellulose with high specificity, has been used to visualize bundles of cellulose bundles in the cell wall of Arabidopsis root epidermis cells [6]. With a confocal microscope, cellulose bundle orientation could be followed over time, providing the first direct proof of passive reorientation of cellulose bundles during cell elongation. Nevertheless, only the largest cellulose bundles could be visualized, as the resolution of conventional light microscopy is limited to around $250 \mathrm{~nm}$ in xy-direction. Cellulose microfibrils have a diameter below $10 \mathrm{~nm}[7,8]$, but can be bundled into macrofibrils. Destructive methods that offer resolution on the $\mathrm{nm}$ scale such as scanning electron microscopy and atomic force microscopy indicate that macrofibrils do not necessarily show the same orientation as the majority of microfibrils $[9,10]$.

In order to achieve a more complete and dynamic understanding of the structure of the cellulose network in plant cell walls a resolution increase for light microscopy is required that allows showing the cell wall in its native state and its differentiation in living cells. The extensive preparation of invasive high resolution techniques, such as scanning electron microscopy (SEM) and atomic force microscopy (AFM) are not compatible with live microscopy and were discussed to have profound impact on the cellulose network structure $[9,11]$.

Recent developments in the field of light microscopy significantly increased the resolving capacity of fluorescence microscopes following different approaches (reviewed by Schermelleh et al. [12]). Basically, these approaches improve resolution by circumventing the diffraction-limits of microscopes as defined by Ernst Abbe [13]. We used two of these so-called super-resolution microscopy techniques, 3D-structured illumination microscopy (3D-SIM) and direct stochastic optical reconstruction microscopy (dSTORM), to test visualization of PFS-stained cellulose fibrils. The third of the established super-resolution techniques, stimulated emission depletion microscopy (STED) is not compatible with green plant tissues, since the depletion laser wavelength is highly absorbed by chlorophyll in the sample. In comparison to wide field fluorescence microscopes, 3D-SIM and STORM improve the resolution of fluorescent signals by, respectively, 2 fold in xyz and by up to 10 fold in xy [12]. In addition, we also tested the effects of deconvolution on confocal image stacks which leads to clearer micrographs by removing the distorting effects of the point-spread function. This function is an inherent factor of any optical system and can be compensated by appropriate algorithms.

Onion bulb scale epidermis, which is used in the present study, has been identified as an ideal model system for the investigation of how cells control growth [14]. Knowing the exact structure of the cellulose network in the cell wall is therefore of immediate significance.

\section{Results and discussion}

\section{Spectral properties and cell toxicity of Pontamine Fast} Scarlet in situ

A simultaneous excitation and emission scan on PFSstained onion bulb scale epidermis cell walls shows a broad excitation range from 485 to $565 \mathrm{~nm}$ (Figure 1). It is therefore well suited for use with the popular $488 \mathrm{~nm}$ and $561 \mathrm{~nm}$ laser lines. The results mirror earlier measurements performed in vitro $[6,15]$. The emission maximum was detected in the red spectrum with a broad peak around $615 \mathrm{~nm}$ (Figure 1). It can therefore readily be distinguished from GFP, YFP, FITC and other commonly used fluorophores that are excited at $488 \mathrm{~nm}$. The partial overlap of the emission spectra of chlorophyll autofluorescence and PFS fluorescence is in most cases not a problem because chloroplasts and cell walls are easily distinguished.

The viability of the plant cells after staining with PFS was tested with carboxyfluorescein-diacetate (CFDA) [16]. The nonpolar CFDA molecules are able to pass the plasma membrane. In the cytoplasm, they gain their fluorescent

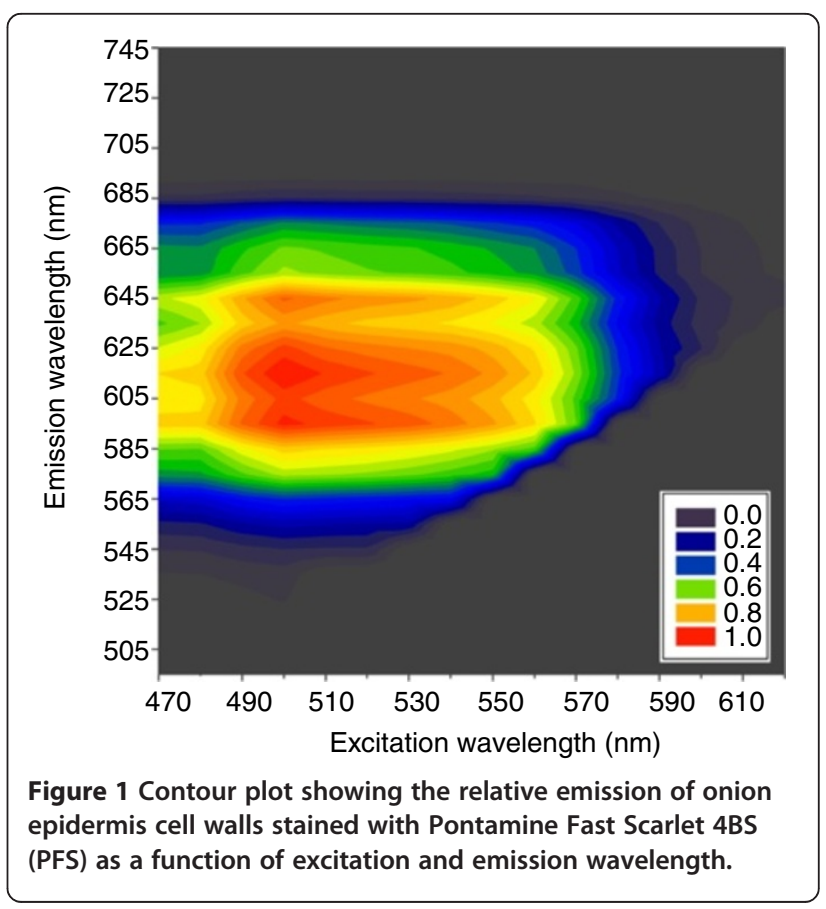


properties through cleavage by endogenous esterases. Intact cells therefore show a green signal in the cytoplasm, where CFDA accumulates. The absence of esterase activity in dead cells prevents the activation of the fluorescence. In confocal microscopy (Figure 2), the CFDA signal could be observed in the cytoplasm of onion bulb scale epidermis cells even after 3 hours of staining with PFS. This did not change with longer incubation times (data not shown), demonstrating the potential of PFS as a general cell wall stain in long-term live-cell experiments. Propidium Iodide, the most common general cell wall dye with emission in the red spectrum, in contrast, is mutagenic, posing a health threat for sample and scientist [16].

\section{Direct stochastic optical reconstruction microscopy shows PFS-stained cellulose fibrils on the cell surface}

In direct STORM (dSTORM), fluorochromes can be switched between a fluorescing and a dark state when using an appropriate excitation wavelength and intensity. Due to the stochastic character of the fluorescent state, it is recorded as blinking and allows separation of neighboring fluorophore molecules in time. If a sufficient number of photons during single blinking event is recorded, the resolution can be improved down to $30 \mathrm{~nm}$ [17]. This method can be used most efficiently with photo-switchable fluorophores, but it has been shown that dSTORM is also possible with conventional dyes, based on their inherent switching capacity [18]. A pre-requisite for dSTORM is that the excitation is limited to a narrow optical section to exclude localization uncertainty that would be present in a large $\mathrm{z}$-volume. This is generally achieved by total internal reflection (TIRF) microscopy. After PFS-staining, cellulose fibrils could be visualized in the cell walls of onion bulb scale epidermis cells using TIRF (Figure 3A). After bleaching with strong excitation laser light, it was furthermore possible to reduce the PFS fluorescence signal so far that single blinking events could be recorded. Figure 3B shows that the rendering of superresolution images based on the localization results, yields a significant gain in resolution.
Fundamentally, two factors are important for a good dSTORM dye: 1) The number of photons detected per dye molecule. This will determine the precision by which the dye molecules are localized. 2) The on-off duty cycle. Dempsey et al. defines the on-off duty cycle as: "the fraction of time a fluorophore spends in the on state". Even with perfectly localized dye molecules the duty cycle will influence the resolution of structures [19]. In regard to these factors, PFS is not an ideal probe for dSTORM. The observed blinking events suggest that PFS, when bound to cellulose, generally has only a few switching cycles before irreversible photodestruction and has a long dark state, meaning that the duty cycle is very low. In addition, during the on-time of 5-10 ms only a relatively low amount of photons can be detected. In practice, this means that a large number of images have to be acquired in order to get a complete super-resolution image.

The method might be improved by employing an imaging buffer containing an oxygen-scavenging system and a primary thiol as reducing compound. Reducing conditions stabilize the off-state of fluorophores, thus preventing their irreversible photodestruction. At the same time, the buffer conditions induce spontaneous recovery of fluorophore molecules to their on-state. Optimization of thiol concentration and excitation laser intensity could lead to an increased number of localizations per frame and duty cycles. This should result in faster imaging and higher resolution [20].

The cellulose fibrils in Figure $3 \mathrm{~B}$ have a diameter of around $100 \mathrm{~nm}$ with clear spacing between them and appear to branch at various positions. This structure on the cell surface can be significantly different from inner layers, which are generally expected to show narrower-spaced, more uniform fibrils [9]. Nevertheless, the observed form and pattern reflect the structural changes achieved during cell expansion and can, as such, give indications of how this process happens. The dSTORM images obtained here could point to the possibility of increased aggregation of fibrils during restructuring, although data from other cell-wall layers obtained with a similar method would be necessary for comparison.

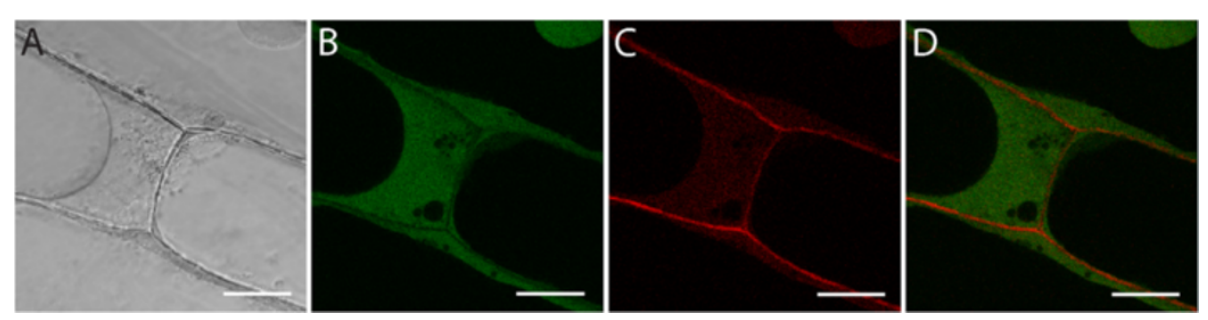

Figure 2 Confocal micrographs of the onion bulb scale epidermis co-stained with PFS and Carboxyfluorescein-Diacetate (CFDA) for $4 \mathrm{~h}$ show non-toxicity of PFS. (A) Bright field image. (B) CFDA fluorescence. (C) PFS fluorescence. (D) Overlay of B and C. Scale bars $50 \mu \mathrm{m}$. 

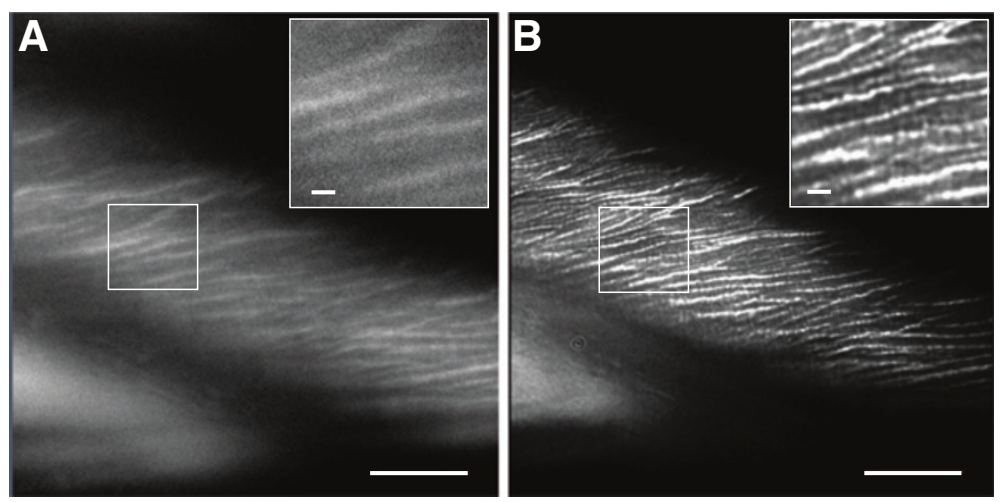

Figure 3 Super-resolution images of PFS-stained cellulose fibrils in onion bulb scale epidermis cells. (A) Total internal reflection fluorescence (TIRF) image. (B) Rendering of stochastic optical reconstruction microscopy (STORM) results. Scale bars $10 \mu \mathrm{m}, 1 \mu \mathrm{m}$ in inserts.

\section{PFS is not suited for visualization of cellulose with 3D structured illumination}

In 3D-SIM, patterned excitation light is used to take images at various phases, rotations and focus planes. From the resulting diffraction patterns, an image with higher resolution than wide-field images can be obtained via mathematical reconstruction in the Fourier space. Usually, this technique is well suited for imaging in plant tissue as it works with conventional probes and moderately thick specimens $[21,22]$. It was, however, not possible to resolve cellulose fibrils in the outer cell wall of onion bulb scale epidermis cells clearly. The Additional file 1: Figure S1 shows a comparison of the PFS-stained outer wall epidermis in wide field and 3D-SIM mode with two different noise filter settings. While standard noise filtering results in loss of any structural information, minimal noise filtering results in typical rotation-symmetric 3DSIM artefacts as seen in the center of Additional file 1: Figure S1. Only small parts of the image reveal a fibril-like structure. It cannot be ruled out that strongly ordered cell wall macrofibrils from other cell types, like sclerenchyma fibers, would be resolved by 3D-SIM. However, discrimination of artefacts from real structures appears difficult - in any case for the example used here.

The effects of different filter settings indicate that the background signal level in PSF-stained onion cells is too high. In this respect the 3D-SIM mode behaves like a widefield microscope rather than a confocal microscope, where out-of focus fluorescence is strongly reduced already under recording.

\section{Confocal imaging combined with image deconvolution can show the cellulose fibril network in 3D}

While not usually considered as a super-resolution technique, image deconvolution, a mathematical operation to recover signal from blurring and noise, can also be used to enhance the resolution capacity of conventional microscopes beyond the diffraction limit. In comparison to STORM and 3D-SIM the gain in resolution is modest, but image deconvolution has the advantage of being applicable to confocal image data. This means that no special imaging hardware is required and that structures deep inside the specimen can be imaged due to the optical sectioning capacity of confocal microscopes.

Data can be acquired with point-scanning, as well as with spinning disc confocal systems. We used a spinning disc confocal, which offers a higher acquisition rate and lower photobleaching than a point confocal. After acquiring an image stack it is imported into adequate image processing software, of which the open source ImageJ and the commercial Huygens SVI are considered to give the best results [23]. The critical factor for deconvolving images is the point spread function, which can be determined experimentally or theoretically. We chose theoretical calculation as the determination of experimental point-spread functions can be unreliable for thick specimen, like whole-tissue samples [24]. Nevertheless, improvement in deconvolution efficiency might be realized if reference objects at the actual focal plane, for example fluorescent beads attached to the outside of a cell, are included during imaging and then used to calculate the sample-inherent point spread function.

Application of deconvolution on image stacks of PFSstained cellulose in the cell wall of onion epidermis cells improved image quality, providing a clearer view of cellulose fibril orientation (Figure 4) and cellulose depositions around plasmodesmata (Figure 5). The raw data shows that, similar to the situation in Arabidopsis root epidermis cells [6], the orientation of cellulose fibrils can be deduced from relatively large bundles, both at the outer (Figure 4A) as well as at the inner side (Figure 4C) of the cell wall. While the deconvolved images show principally the same fibrils, the enhanced resolution 
enables clear distinction of single fibrils (Figure 4B,D). This is essential for accurate quantification, for example of the angle between fibrils and the longitudinal axis of the cell. Measuring 20 fibrils, an average angle of $46.2^{\circ}$ (standard error $3.1^{\circ}$ ) was determined at the focal plane $100 \mathrm{~nm}$ below the cell surface, while 20 fibrils $300 \mathrm{~nm}$ deeper inside the cell wall are arranged at $\mathrm{n}$ average angle of $92^{\circ}$ (standard error $1.8^{\circ}$ ). Two distinct layers with different cellulose orientation reflects different cell expansion phases, in which cellulose in the outer layer reorients passively after xyz deposition as proposed in the passive reorientation hypothesis [25]. This information can complement onion epidermis growth studies that only analyze the mean cellulose orientation in the whole cell wall $[3,14]$.

Taken the optical resolution of some 200 by $550 \mathrm{~nm}$ (x by $\mathrm{z})$ with the objective lens applied (N.A =1.4) at the emission maximum of PFS $(600 \mathrm{~nm})$, the observed change of fibril orientation at a focal distance of $200 \mathrm{~nm}$ appears impossible. However, the strength of deconvolution lies exactly in its power to improve the 3-D resolution by removing the blurring effects of the zeppelin-shaped pointspread function. This technique would not allow to separate two points in the specimen lying on top of each other on the optical axis (= $z$-axis) within this distance, but is able to do so for the linear structures as the macrofibrils in the sample used here. This means that two microfibrils running parallel on top of each other with a distance of $200 \mathrm{~nm}$ in $z$ cannot be separated, but those which are crisscrossing. Here the deconvolution algorithm is able to improve the contrast between the fiber in focus and the gap (no fluorescence) $200 \mathrm{~nm}$ below sufficiently for visualization.

In addition to cellulose orientation, deconvolution of images of PFS-stained onion epidermis cell wall also reveals features of the cell connection architecture (Figure 5). While raw data of co-staining with the callose-specific dye Anilin Blue suggests incongruent distribution domains of cellulose in callose in the cell wall (Figure 5A,B), this becomes much clearer on deconvolved images (Figure 5C, D), even showing the thin cell wall plate that remains in the pit fields. Interestingly, in 3D view or maximum intensity projections of deconvolved images, it becomes apparent that cellulose is deposited at much higher density in areas of $2 \mu \mathrm{m}$ around callose compared to the rest of the wall (Figure 5F). This cellulose collar could potentially form a limit to the callose deposits that are generally enhanced by wounding in order to plug the plasmodesmata [26].
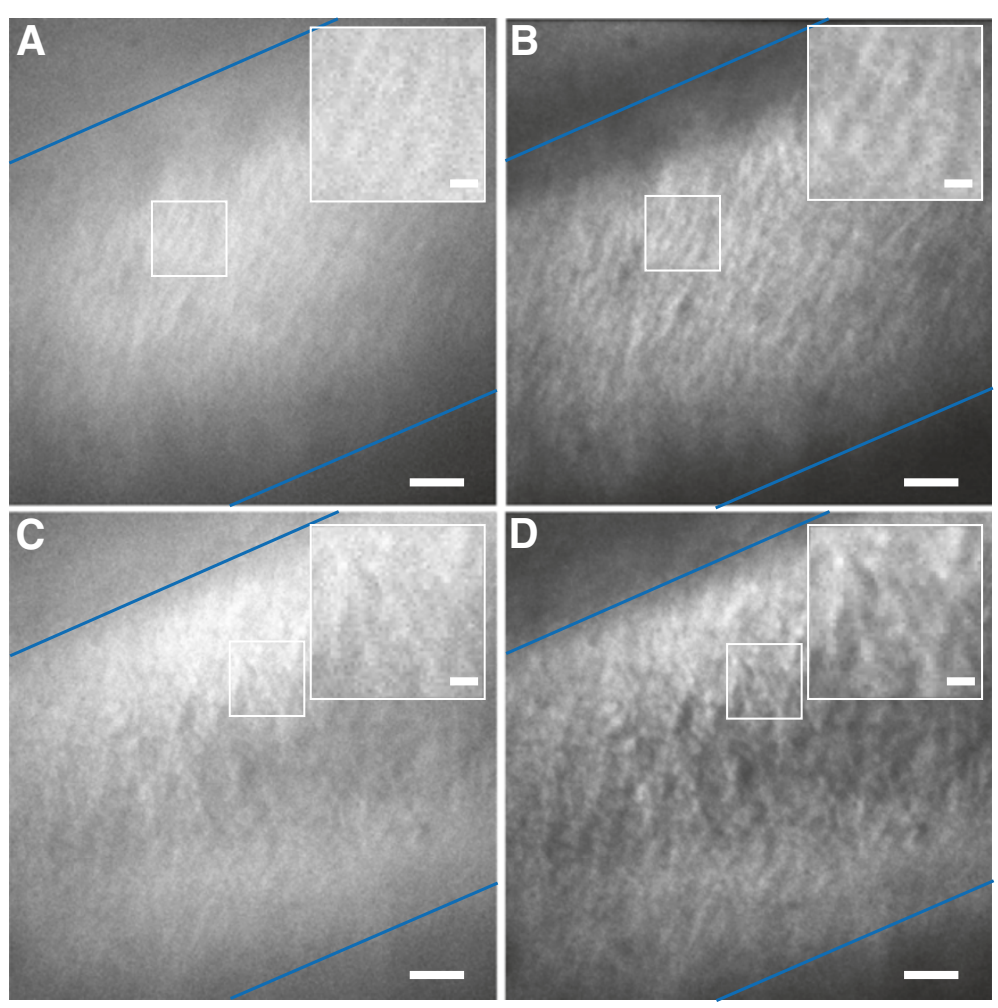

Figure 4 PFS-stained cellulose fibril orientation imaged with conventional confocal microscopy $(A, C)$ and in combination with post-acquisition image deconvolution (B, D); Cellulose fibrils are at an angle close to $45^{\circ}$ at a focal plane about $100 \mathrm{~nm}$ below the outer cell wall surface (A, B) compared to an angle around $90^{\circ}$ at a focal plane about $450 \mathrm{~nm}$ below the outer cell wall surface (C, D); Blue lines indicate borders to neighboring cells. Scale bars $10 \mu \mathrm{m} ; 2 \mu \mathrm{m}$ in inserts. 


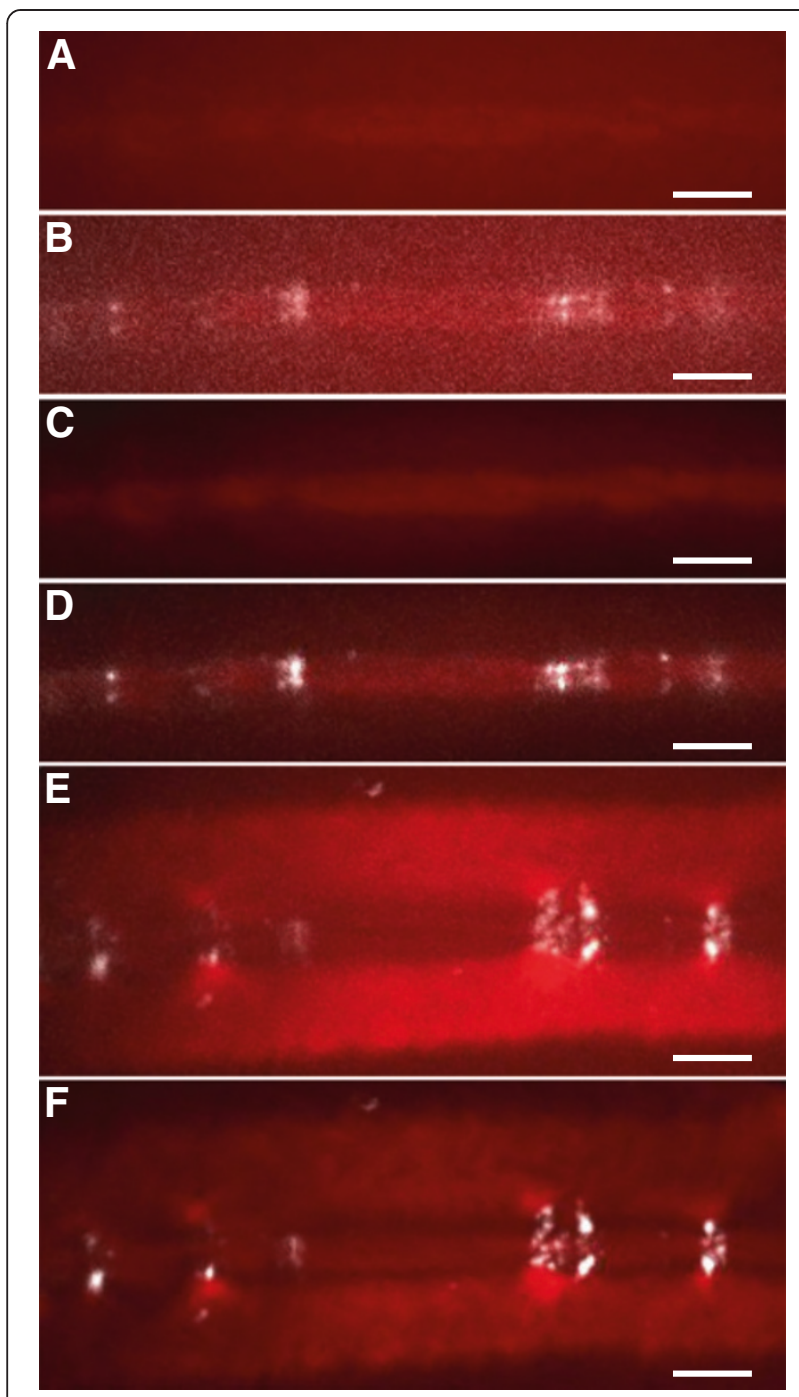

Figure 5 Cellulose and callose around cell connections imaged with conventional confocal microscopy (A, B, E) and in combination with post-acquisition image deconvolution (C, D, F); a raw confocal image of PFS-stained cellulose in the cell wall between neighboring cells, $b$ overlay with image of Anilin Blue-stained callose; After deconvolution the same images (C, D) show more clearly that callose and cellulose domains do not overlap; in maximum intensity projections a higher cellulose concentration can be clearly located around the callose depositions after deconvolution (F) compared to raw confocal images (E). Scale bars $10 \mu \mathrm{m}$.

\section{Conclusion}

Wide-field fluorescence microscopy allows investigating cell walls in their native state, but further details of its substructure can be uncovered when improving the microscopic resolution power. Here we demonstrate that the cellulose-specific dye PFS is suited for use in TIRF microscopy and STORM, realizing the resolution of cellulose fibrils below $100 \mathrm{~nm}$ thickness. Nevertheless, since TIRF microscopy limits observation to the cell surface, no information can be gained from deeper cell wall layers. While
3D-SIM principally enables super-resolution imaging even more than $100 \mu \mathrm{m}$ inside a biological specimen, the high signal density of PFS in the cell wall prevented a correct mathematical reconstruction of the fibrillar substructure in the onion epidermis. As an alternative to STORM and 3D-SIM we used conventional confocal microscopy with post-acquisition image deconvolution. This enables a more modest increase in resolution compared to the other approaches, but leverages the z-sectioning and deep imaging capacity of confocal microscopy. This approach yielded data that enabled us to assess the orientation of cellulose fibrils, which was different in two layers within the cell wall. It was also used in co-staining experiments with a dye for another cell wall component, which revealed new features of cell wall architecture around plasmodesmata between neighboring cells.

Future developments, like the adaptation of 3D-STORM [27] for plant tissues will, without doubt, help to further close the resolution gap between non-invasive light microscopy and invasive techniques like AFM and SEM. The results presented here show how a first step towards this goal can be realized with the super-resolution techniques that are currently available, demonstrating their potential to be a valuable tool for the investigation of cell wall structure of living plant cells at a resolution higher than what had been technically possible before.

\section{Methods}

\section{Dyes and staining}

Pontamine Fast Scarlet 4 BS was purchased from Aldrich Rare Chemicals Library (catalog no. S479896; SigmaAldrich, St. Louis, USA). It is now available under the synonym Direct Red. Stock solutions of $5 \mathrm{mg} / \mathrm{ml}$ in $0.1 \mathrm{M}$ phosphate buffered saline (PBS) pH 7.2 can be stored at $-4^{\circ} \mathrm{C}$ or $-20^{\circ} \mathrm{C}$ for several weeks. The working solution was diluted to $0.1-0.01 \mathrm{mg} / \mathrm{ml}$ depending on the tissue and was always prepared fresh. Incubation time was usually $20 \mathrm{~min}$, but longer in complex tissues. Staining was followed by brief washing in PBS.

Carboxyfluorescein-diacetate (Molecular Probes, Life Technologies Corporation, Carlsbad, USA) was used at $0.5 \mu \mathrm{g} / \mathrm{ml}$ in PBS. Tissue was stained in 10 to 15 minutes, followed by 5 minutes washing in phosphate buffer.

Aniline Blue (Sigma-Aldrich, St. Louis, USA) working solution was diluted to $50 \mu \mathrm{g} / \mathrm{ml}$ in PBS and staining time was 5 to 10 minutes.

\section{Sample preparation}

Onion bulb scale epidermis was prepared from Allium cepa bulbs purchased in a supermarket. Using a razorblade $0.5 \times 0.5 \mathrm{~cm}$ pieces were cut out from fresh scale and the epidermis peeled off from the inner side with tweezers. 


\section{Spectral analysis}

A simultaneous excitation and emission scan (Lambda ${ }^{2}$ scan) was performed in situ on onion bulb scale epidermis cell walls stained with PFS using a Leica SP5-X confocal laser-scanning microscope (Leica Microsystems, Mannheim, Germany). A white light laser, calibrated to keep a constant output power over the available spectrum, was used as excitation source. Excitation scans were made every $10 \mathrm{~nm}$ between $470 \mathrm{~nm}$ and $620 \mathrm{~nm}$. The AOBSdefined detection window of $10 \mathrm{~nm}$ width was shifted from excitation wavelength plus $15 \mathrm{~nm}$ (center position) to $745 \mathrm{~nm}$ for each excitation wavelength. Image analysis was performed in ImageJ.

\section{Image acquisition and processing}

For confocal imaging in the cell vitality test a Leica TCS SP2/MP (Leica Microsystems, Mannheim, Germany) was used. PFS and cFDA were excited by an Argon Laser at $488 \mathrm{~nm}$. The following detection windows were defined: PFS: 560-605 nm, cFDA: 485-505 nm.

Super-resolution imaging was performed on a Zeiss Elyra PS.1 microscope (Zeiss, Jena, Germany) and an Andor iXion 860 EMCCD camera (Andor Technology, Belfast, Ireland) using a $561 \mathrm{~nm}$ solid state laser for excitation, a band pass filter with DM 561 nm, EM 570-650 nm.

For 3D-SIM $63 \times 1.2$ Zeiss Apochromat water-immersion and $63 \times 1.4$ Zeiss Apochromat oil-immersion objectives were used together with a $23 \mu \mathrm{m}$ grating. Z-stacks were recorded with 5 phase-changes and 5 grating rotations for each section. The 3D-SIM wizard in the Zeiss Zen 2011 software was used for image processing with the following setting: SR frequency weighing 1, Baseline cut, theoretical PSF and noise filtering as specified in the image legend.

For STORM, the same microscope was used with a $100 \times 1.46$ Zeiss Apochromat oil-immersion objective. The TIRF angle was adjusted by focusing on the surface of the onion epidermis cells in epi-fluorescence mode, then switching to TIRF-mode and increasing the angle until cellulose fibers became visible (around $60^{\circ}$ ). For one image series, 20000 images were acquired. These were processed inside the Zeiss Zen 2011 software using the PALM-wizard. The filter settings were chosen permissive compared to settings generally used when working with photoswitchable proteins with relatively low chi-square and background thresholds. False positives were filtered out by setting the minimum photon count to 100 . From the vector map of localization precisions, the background-corrected point-spread functions were rendered with a localization precision of $75 \mathrm{~nm}$. The on time and dark state interval of PFS fluorophores were determined in separate image series. Only a $128 \times 128$ pixel area of the camera sensor was read out in order to enable image acquisition times of about $3 \mathrm{~ms}$ per image.
Deconvolution of confocal images was done after recording optical sections with a spinning-disc confocal (Andor Revolution XD, $100 \times 1.4$ Olympus UPlanSApo objective, Andor iXion 897 EMCCD camera, $561 \mathrm{~nm}$ solid state laser, EX $564 \mathrm{~nm}$, DM 561 nm, EM 570-650 nm). Deconvolution was performed in Image J using the plugins PSF Generator and DeconvolutionLab, both developed by Biomedical Imaging Group at the École Polytechnique de Lausanne [28]. With PSF Generator, theoretical point spread functions were generated with the Born and Wolf algorithm [29], which takes account of numerical aperture, refractive indices, wavelength, pixel size and z-step spacing. The resulting point-spread function was used in the deconvolution algorithm developed by Richardson and Lucy [30] with 20 iterations. This Bayesian-based method is generally considered among the most appropriate for deconvolution of confocal data [24]. The output data is compared to raw data without further image adjustments.

\section{Additional file}

Additional file 1: Figure S1. 3D-structured illumination of PFS-stained cellulose fibrils in onion bulb scale epidermis cells; On the wide-field image (a) a high background fluorescence intensity in relation to the in-focus area is obvious. On the same image after processing with standard noise filtering (b) all structural information is lost. Using minimal noise filtering instead (c), artifacts cover most of the in-focus area. Some high-resolution structural information might be preserved (box, same area magnified in insert). Scale bars $10 \mu \mathrm{m} ; 1 \mu \mathrm{m}$ in insert.

\section{Competing interests}

The authors declare that they have no competing interests.

\section{Authors' contributions}

$J$ conceived and designed the study, performed experiments and wrote the manuscript. AS assisted with experiment design and discussed analysis and interpretation of image data and the manuscript. IZ assisted with

interpretation of super-resolution image data and image processing.

All authors read and approved the final manuscript.

\section{Acknowledgements}

All microscopy was performed at the Center for Advanced Bioimaging (CAB) Denmark. We thank Michael Hansen, University of Copenhagen, for advice on super-resolution image processing.

Received: 23 October 2013 Accepted: 23 December 2013 Published: 28 December 2013

\section{References}

1. Baskin TI: Anisotropic expansion of the plant cell wall. Ann Rev Cell Dev Biol 2005, 21:203-222.

2. Geitmann A: Mechanical modeling and structural analysis of the primary plant cell wall. Curr Opin Plant Biol 2010, 13:693-699.

3. Suslov D, Verbelen JP: Cellulose orientation determines mechanical anisotropy in onion epidermis cell walls. J Exp Bot 2006, 57:2183-2192.

4. Himmel ME, Ding SY, Johnson DK, Adney WS, Nimlos MR, Brady JW, Foust TD: Biomass recalcitrance: engineering plants and enzymes for biofuels production. Science 2007, 315:804-807.

5. Keegstra K: Plant cell walls. Plant Phys 2010, 154:483-486.

6. Anderson CT, Carroll A, Akhmetova L, Somerville C: Real-time imaging of cellulose reorientation during cell wall expansion in Arabidopsis roots. Plant Phys 2010, 152:787-796. 
7. Ha MA, Apperley DC, Evans BW, Huxham IM, Jardine WG, Vietor RJ, Reis D, Vian B, Jarvis MC: Fine structure in cellulose microfibrils: NMR evidence from onion and quince. Plant J 1998, 16:183-190.

8. Kennedy CJ, Cameron GJ, Sturcova A, Apperley DC, Altaner C, Wess TJ, Jarvis MC: Microfibril diameter in celery collenchyma cellulose: X-ray scattering and NMR evidence. Cellulose 2007, 14:235-246.

9. Ding SY, Himmel ME: The maize primary cell wall microfibril: a new model derived from direct visualization. J Agr Food Chem 2006, 54:597-606.

10. McCann MC, Bush M, Milioni D, Sado P, Stacey NJ, Catchpole G, Defernez M, Carpita NC, Hofte H, Ulvskov P, et al: Approaches to understanding the functional architecture of the plant cell wall. Phytochemistry 2001, 57:811-821.

11. Sarkar P, Bosneaga E, Auer M: Plant cell walls throughout evolution: towards a molecular understanding of their design principles. J Exp Bot 2009, 60:3615-3635.

12. Schermelleh $\mathrm{L}$, Heintzmann $\mathrm{R}$, Leonhardt $\mathrm{H}$ : A guide to super-resolution fluorescence microscopy. J Cell Biol 2010, 190:165-175.

13. Abbe $\mathrm{E}$ : A contribution to the theory of the microscope and the nature of microscopic vision. Proc Bristol Nat Soc 1874, 1:200-261.

14. Suslov D, Verbelen JP, Vissenberg K: Onion epidermis as a new model to study the control of growth anisotropy in higher plants. J Exp Bot 2009, 60:4175-4187.

15. Hoch HC, Galvani CD, Szarowski DH, Turner JN: Two new fluorescent dyes applicable for visualization of fungal cell walls. Mycologia 2005, 97:580-588.

16. Oparka KJ, Read N: The use of fluorescent probes for studies on living plant cells. In Plant Cell Biology: A Practical Approach. Edited by Harris N, Oparka KJ. Oxford: Oxford University Press; 1994:27-50.

17. Heilemann M, van de Linde S, Schuttpelz M, Kasper R, Seefeldt B, Mukherjee A, Tinnefeld P, Sauer M: Subdiffraction-resolution fluorescence imaging with conventional fluorescent probes. Angew Chem Int Edit 2008, 47:6172-6176.

18. Shim SH, Xia CL, Zhong GS, Babcock HP, Vaughan JC, Huang B, Wang X, Xu C, Bi GQ, Zhuang XW: Super-resolution fluorescence imaging of organelles in live cells with photoswitchable membrane probes. Proc Natl Acad Sci U S A 2012, 109:13978-13983.

19. Dempsey GT, Vaughan JC, Chen KH, Bates M, Zhuang X: Evaluation of fluorophores for optimal performance in localization-based super-resolution imaging. Nat Methods 2011, 8:1027-1036.

20. Vogelsang J, Cordes T, Forthmann C, Steinhauer C, Tinnefeld P: Controlling the fluorescence of ordinary oxazine dyes for single-molecule switching and superresolution microscopy. Proc Natl Acad Sci USA 2009, 106:8107-8112.

21. Fitzgibbon J, Bell K, King E, Oparka K: Super-resolution imaging of plasmodesmata using three-dimensional structured illumination microscopy. Plant Phys 2010, 153:1453-1463.

22. Linnik O, Liesche J, Tilsner J, Oparka KJ: Unraveling the structure of viral replication complexes at super-resolution. Front Plant Sci 2013, 4:6

23. Griffa A, Garin N, Sage D: Comparison of deconvolution software in 3D microscopy. A user point of view. GIT Imaging Microsc 2010, 1:41-43.

24. de Monvel JB, Le Calvez S, Ulfendahl M: Image restoration for confocal microscopy: improving the limits of deconvolution, with application to the visualization of the mammalian hearing organ. Biophys J 2001, 80:2455-2470

25. Chan J: Microtubule and cellulose microfibril orientation during plant cell and organ growth. J Microsc 2012, 247:23-32.

26. Roberts A, Oparka K: Plasmodesmal structure and development. In Plasmodesmata. Edited by Oparka K. Oxford: Blackwell Publishing; 2005:1-31. Annual Plant Reviews.

27. Huang B, Jones SA, Brandenburg B, Zhuang XW: Whole-cell 3D STORM reveals interactions between cellular structures with nanometer-scale resolution. Nat Methods 2008, 5:1047-1052.
28. EPFL Biomedical imaging group algorithms. http://bigwww.epfl.ch/ algorithms.html.

29. Born M, Wolf E: Principles of Optics. 7th edition. Cambridge: Cambridge University Press; 2002.

30. Richardson W: Bayesian-based iterative method of image restoration. J Opt Soc Am 1972, 62:55.

doi:10.1186/1471-2229-13-226

Cite this article as: Liesche et al: Super-resolution imaging with Pontamine Fast Scarlet 4BS enables direct visualization of cellulose orientation and cell connection architecture in onion epidermis cells. BMC Plant Biology 2013 13:226.

\section{Submit your next manuscript to BioMed Central and take full advantage of:}

- Convenient online submission

- Thorough peer review

- No space constraints or color figure charges

- Immediate publication on acceptance

- Inclusion in PubMed, CAS, Scopus and Google Scholar

- Research which is freely available for redistribution

Submit your manuscript at www.biomedcentral.com/submit
C) Biomed Central 